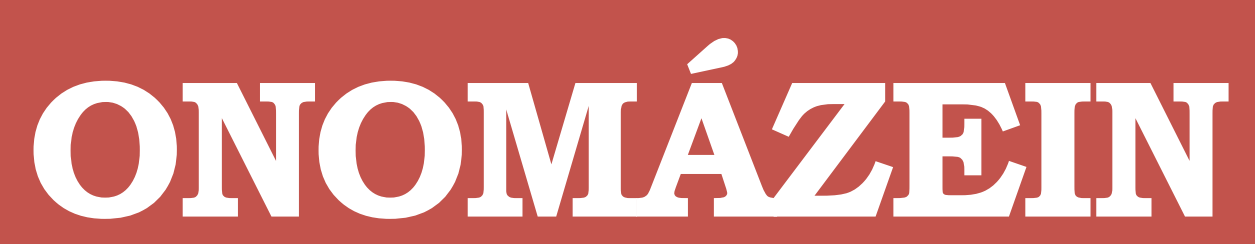

Revista de lingüística, filología y traducción
PONTIFICIA UNIVERSIDAD

\title{
¿Coordinadores discontinuos en español? Problemas de análisis de los correlativos disyuntivos y copulativos*
}

Initial coordination in Spanish?

Problems of analysis of the disjunctive

and copulative correlatives

\section{Carmen Conti Jiménez}

Universidad de Jaén

España

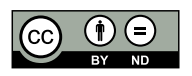

Carmen Conti Jiménez: Departamento de Filología Española, Universidad de Jaén, España.

| E-mail: cconti@ujaen.es 


\section{Resumen}

En este artículo se pretende demostrar que la primera partícula de las secuencias o... o y ni... ni del español, que se ha analizado tradicionalmente como coordinador o parte de un coordinador discontinuo, muestra pautas de comportamiento gramatical, pragmático y prosódico propias de las partículas correlativas focales de otras lenguas. Con este fin, este artículo, que se basa en el análisis de ejemplos extraídos del Corpus del Español del Siglo XXI para el español europeo, gira en torno a dos ejes fundamentales: de una parte, la presentación de los patrones distribucionales y de combinatoria que desaconsejan el análisis de estas formas como coordinadores y, de otra, la demostración de que tanto esos patrones como otros adicionales son prueba de la naturaleza correlativa y focal de estas partículas.

Palabras clave: coordinadores discontinuos; partículas focales; correlativos.

\section{Abstract}

This paper examines disjunctive and copulative initial coordination in Spanish $\left(O_{1} \ldots O_{2}\right.$ 'either... or' and $n i_{1} \ldots n i_{2}$ 'neither... nor') with a view to demonstrating that, in contrast to conventional analyses, the former element does not pass the proofs of the coordinating conjunctions. To do so, we will base our proposal on the grammatical patterns observed in a compilation of examples from the "Corpus del Español del Siglo XXI" (in particular, from written texts in European Spanish). These patterns allow us to suggest that $O_{1}$ and $n i_{1}$ are not coordinators, but focal particles whose scope or dominion is the first conjunct coordinated by $\mathrm{O}_{2}$ or $\mathrm{ni}_{2}$.

Keywords: initial coordination; focal particles; correlative particles.

* Este artículo forma parte de la investigación realizada en el marco del proyecto Cosubordinación en español (ref. FFI2017-85429-P), del Ministerio de Ciencia, Innovación y Universidades del Gobierno de España. 


\section{Introducción}

Los trabajos descriptivos sobre la oración compuesta en español tipifican las construcciones con o... o y ni... ni de (1) dentro de la coordinación, atendiendo fundamentalmente al análisis de estas partículas como conjunciones coordinantes o coordinadores. A partir de la monografía de Franchini (1986), no es infrecuente referirse a estos elementos como conjunciones o coordinadores discontinuos, en oposición a las conjunciones simples:

(1) a. $\mathrm{O}_{1}$ estudias $\mathrm{O}_{2}$ trabajas.

b. $\mathrm{Ni}_{1}$ estudia $\mathrm{ni}_{2}$ trabaja.

En este artículo se pretende demostrar, sin embargo, que las estructuras con o... o y ni... ni presentan un comportamiento gramatical distinto del atribuido a las construcciones coordinadas. Para su estudio, se ha partido del análisis de una selección de ejemplos extraídos del Corpus del Español del Siglo XXI (RAE) para el español europeo escrito (v. § 3 sobre metodología). El análisis de los datos permite constatar que, en efecto, $O_{1}$ y $n i_{1}$ no tienen la función de coordinar segmentos: como se mostrará en la sección 4, estas partículas, a diferencia de los coordinadores, pueden aparecer precedidas por la conjunción y, ocupan siempre una posición antepuesta al primer segmento coordinado y presentan restricciones de combinatoria de las que carecen y/o. Además, $o_{1}$ y $n i_{1}$ condicionan la estructura informativa de la oración, lo que pone de manifiesto que desempeñan una acusada función pragmática.

Asimismo, en este artículo se pretende demostrar que las propiedades gramaticales, pragmáticas y prosódicas de $O_{1}$ y $n i_{1}$ responden a las pautas de comportamiento de los llamados correlativos, una macroclase de palabras con relevancia pragmática (v. § 5$) \cdot O_{1}$ y $n i_{1}$, al igual que los correlativos, exigen un correlato formalmente condicionado $\left(\mathrm{O}_{2}\right.$ y $\left.n i_{2}\right)$, no son recursivos (solo pueden aparecer una vez), preceden siempre al correlato y solo se combinan con proyecciones máximas o sintagmas. En lo que respecta a su función pragmática, $\mathrm{O}_{1}$ y $n i_{1}$, como sucede con los correlativos disyuntivos y copulativos de otras lenguas ( $v$. el inglés either 'o' y neither 'ni'), muestran rasgos que los aproximan a las partículas focales: abren una curva prosódica de anticadencia o semianticadencia tonal que se cierra en el correlato $\left(O_{2}\right.$ y $\left.n i_{2}\right)$, expresan exclusión y condicionan la codificación del tópico.

Las partículas $\mathrm{O}_{1}$ y $n i_{1}$, al igual que otros correlativos focales, suponen un reto para el análisis de constituyentes. Esto se debe, sobre todo, al hecho de que los correlativos, sean o no resultado de un doblado formal, presentan numerosos problemas de adscripción categorial y difícilmente se pueden interpretar como núcleos de una proyección máxima. Si bien en este artículo no se pretende ofrecer una solución a este problema, en la sección 6 se mostrará la conveniencia de tratar $\mathrm{O}_{1}$ y $n i_{1}$ como operadores de foco con repercusión sobre la fuerza ilocutiva de la cláusula. 
Hecha esta breve presentación, pasamos a enumerar las secciones de las que se compone este artículo. En la sección 2 se revisan los distintos tratamientos que han recibido o... o y ni... ni en la bibliografía previa. En la sección 3 se resumen algunas cuestiones relacionadas con la obtención y el tratamiento de los ejemplos empleados. En la sección 4 se muestran los problemas que surgen del análisis de $O_{1}$ y $n i_{1}$ como coordinadores, para lo cual se atenderá a factores como la aparición de estas partículas con la conjunción $y$, sus restricciones distribucionales y de posición. En la sección 5 se ofrece una propuesta de análisis de $O_{1}$ y $n i_{1}$ como partículas correlativas focales basada en sus propiedades pragmáticas, semánticas y prosódicas y en los paralelismos observados entre $O_{1}$ y $n i_{1}$ y las partículas correlativas de algunas lenguas germánicas (como el inglés either... or 'o... o'). En la sección 6 se plantean los problemas que suscitan $O_{1}$ y $n i_{1}$ para el estudio de la formación de constituyentes. Finalmente, en la sección 7 se presentan las conclusiones.

\section{Estudios previos sobre o... o y ni... ni}

Las partículas o... o y ni... ni se han analizado tradicionalmente como conjunciones coordinantes, coordinadores o conectores ${ }^{1}$, esto es, como partículas ${ }^{2}$ que unen segmentos o combinan unidades que no están integradas ni muestran rasgos de dependencia gramatical ${ }^{3}$. En cuanto partículas de unión o conectivas, los coordinadores muestran restricciones de combinatoria que los caracterizan frente a otras partículas nexivas, como se verá en la sección 4.1. A partir de la obra monográfica de Franchini (1986) sobre coordinación copulativa en español, las secuencias o... o y ni... ni se analizan con frecuencia como conjunciones discontinuas o coordinadores discontinuos - así, por ejemplo, en RAE/ASALE (2009) - , pues constan de dos elementos formalmente idénticos a los que se atribuye la misma función. El análisis de estas partículas como conjunciones coordinantes se ha defendido no solo en trabajos descriptivos y funcionales del español, sino también en estudios más recientes de

1 Dicha adscripción se encuentra, entre otros, en RAE (1973), Alarcos (1994), Martínez (1994), Camacho (1999) y RAE/ASALE (2009). Para una revisión sobre el tratamiento historiográfico de estas formas, véase Myre (1992).

2 En este artículo se emplea el término partícula para referir a palabras de diferente adscripción categorial o bien a palabras de difícil adscripción categorial, como es el caso de $O_{1}$ y $n i_{1}$.

3 En este artículo se entiende que la coordinación es un tipo combinatorio simétrico que tiene lugar entre segmentos autónomos que son equiparables desde el punto de vista gramatical, semántico y pragmático. Se adopta aquí un enfoque funcional del concepto (Van Valin y LaPolla, 1997; Van Valin, 2005; Hengeveld y Mackenzie, 2008). Esta simetría se observa, principalmente, en su equivalencia funcional y en su pertenencia al mismo nivel jerárquico de representación estructural (Dik, 1968; Franchini, 1986). Además, se ha señalado que los coordinandos han de ser semánticamente homogéneos (Jiménez Juliá, 1995: 39-40) y, en caso de ser cláusulas, también pragmáticamente congruentes (Dik, 1968). Véanse los trabajos de Conti (2014) y (2016) para una descripción detallada de las propiedades de las cláusulas coordinadas en español. 
corte formalista (Aldaber, 2018). En otra línea interpretativa se encuentran, sin embargo, Bosque (1994) (a quien sigue Sánchez, 1999: 2616-261) y Camacho (2003), quienes se desvían del análisis habitual de estas partículas. En concreto, Bosque (1994) señala que ni es un índice morfológico de concordancia negativa con $n i_{2}$, si bien no establece ninguna conexión entre esta partícula y la disyuntiva $O_{1}$ (véase la sección 4.3 para más detalles); Camacho (2003: 138), por su parte, analiza $\mathrm{O}_{1}$ como un operador distributivo y $\mathrm{O}_{2}$ como núcleo funcional, pero no establece diferencias entre $n i_{1}$ y $n i_{2}$, que considera núcleos funcionales de sendas proyecciones máximas (Camacho, 2003: 140).

Asimismo, los trabajos previos en los que se mencionan o se estudian las estructuras con o... 0 y ni... ni han mostrado un especial interés por las diferencias con o y no... ni, respectivamente. Estas diferencias se atribuyen a menudo a razones de índole estilística (como el afán expresivo de ni... ni o el realce de la disyunción en el caso de o... o, ambos aspectos señalados por Alarcos, 1994: 229 y 330), o bien a razones de naturaleza semántica, como la expresión de una disyunción exclusiva con o... o frente a una disyunción no marcada (exclusiva e inclusiva) con o (Jiménez Juliá, 1986). En muy pocos casos, como se verá más abajo, las diferencias entre o... o y o, de una parte, y entre ni... ni y no... ni, de otra, se han centrado en aspectos gramaticales.

Las diferencias semánticas entre las formas dobles y las simples se han tratado con más detalle en trabajos monográficos sobre coordinación (Jiménez Juliá, 1995), coordinación copulativa (Franchini, 1986), disyunción (Fukasawa, 1985; Jiménez Juliá, 1986), coordinación negativa (Jiménez Juliá, 1984) o distribución (Myre, 1992, 1998; Fornés, 1994, 1996)4. Cabe señalar, sin embargo, que el estudio de ni... ni y o... o es marginal en tales casos, pues aparece siempre supeditado a las formas simples o y ni o a sus posibles paráfrasis, como sucede con ni e y no.

Así, en lo que se refiere específicamente a la disyuntiva discontinua, Fukasawa (1985) señala que $0 . .$. o aparece con menos frecuencia en textos que o (p. 67) y que expresa, a diferencia de la conjunción simple, eventos excluyentes (p. 73). En la misma línea, Jiménez Juliá (1986) considera que $0 . .$. o es el término marcado de la oposición, pues exige siempre la exclusión de uno de los miembros de la coordinación (v. 2a), mientras que o es el término no marcado, pues admite una interpretación exclusiva e inclusiva de los miembros coordinados (v. 2b) (así también Jiménez Juliá, 1995: 85):

4 En los trabajos sobre distribución se debate a menudo la pertinencia de incluir las llamadas distributivas (con ora... ora y formas afines) a las disyuntivas, junto a o... o (Myre, 1992, 1998). Las formas distributivas plantean problemas de interpretación categorial (es decir, no es claro su análisis como conjunciones) y, en consecuencia, también suscitan problemas de análisis relativos a la relación que se establece entre las cláusulas combinadas (coordinación o yuxtaposición). De hecho, estas formas se tratan como palabras léxicas en Alarcos (1994: 317) y en Myre (1998: 31), que las incluyen dentro de la yuxtaposición, mientras que se interpretan como conjunciones coordinantes en la última gramática académica (RAE/ASALE, 2009: 2414) y también en Aldaber (2018). 
(2) (Jiménez Juliá, 1986: 169)

a. Llegó o a las dos o a las tres. (Solo a una u otra hora)

b. Llegó a las dos o a las tres. (A las dos, las tres u otra hora)

En cuanto al tipo de relación combinatoria, en los trabajos citados se asume que los segmentos unidos por o... o están coordinados. Sin embargo, Conti (2018a) defiende que las cláusulas de $0 . .$. o son cosubordinadas, ya que muestran rasgos de dependencia gramatical que no presentan los elementos unidos por la conjunción $0^{5}$.

En lo que respecta a ni, el trabajo de Jiménez Juliá (1984) se centra en demostrar que este nexo no es marca de coordinación negativa en español, ya que no niega la relación de unión, sino los miembros coordinados. En opinión del autor, por tanto, no hay diferencia funcional entre ni (dentro o fuera de la estructura correlativa) y la secuencia y no, dado que la primera se puede conmutar siempre por la segunda (p. ej. No bebe ni fuma / Ni bebe ni fuma / No bebe y no fuma). En un trabajo posterior, Jiménez Juliá (1995: 75) añade como prueba de que ni une elementos negados el hecho de que las secuencias no/ni... ni sean incompatibles con predicados colectivos (p. ej. *Ni Ana ni Luis se encontraron en el metro, frente a Ana y Luis no se encontraron en el metro). En concreto, el autor indica que no/ni... ni es posible "cuando cada miembro realiza una acción paralela a la de los demás y, por tanto, puede individualizarse su polaridad negativa” (Jiménez Juliá, 1995: 75). En lo que respecta a las diferencias, Jiménez Juliá (1984: 229) indica que ni se aleja de y no en el tipo de elementos categoriales que coordina ${ }^{6} \mathrm{y}$ en la exigencia por parte de ni (no así de y no) de que todos los términos coordinados estén negados (p. ej. *Bebe ni fuma frente a Bebe y no fuma).

Bosque (1994), por su parte, se ocupa de las restricciones distribucionales y de combinatoria de ni, como el doblado obligatorio en posición preverbal (p. ej. \{*Luis ni Pedro/ni Luis ni Pedro\}

5 Las unidades cosubordinadas son dependientes, pero no son dependientes desde el punto de vista estructural, ya que no funcionan ni como complementos ni como modificadores (Van Valin y LaPoIla, 1997: 454; Van Valin, 2005: 188; Hengeveld y Mackenzie, 2008: 309). Así, por ejemplo, las cláusulas cosubordinadas en español, a diferencia de las cláusulas coordinadas, que no son dependientes, comparten el mismo operador de fuerza ilocutiva, lo que explica que presenten siempre la misma modalidad (Conti, 2018a). Además, las cláusulas de o... o, a diferencia de las cláusulas coordinadas, rechazan el modo imperativo, lo que apunta a su naturaleza dependiente y defectiva. Este mismo comportamiento es extensible a las cláusulas unidas por ni... ni, también tipificadas tradicionalmente dentro de la coordinación. Por razones de espacio y por conveniencia explicativa, no se abordará en este artículo el estudio del tipo de relación combinatoria que expresan estas estructuras.

6 Si bien Jiménez Juliá (1984: 229) no explicita a qué se refiere con esta diferencia categorial, cabe imaginar que está aludiendo al hecho de que no en la secuencia y no solo se combina con cláusulas, mientras que ni puede combinarse con cláusulas y también con constituyentes menores (p. ej. Ni Juan ni Pedro pudieron estudiar). Véase la sección 4.2 para las restricciones combinatorias de los correlativos objeto de estudio. 
están de acuerdo con esa idea) o su agramaticalidad con términos de preposición (p. ej. No sé nada de *ni Juan ni Pedro / No sé nada ni de Juan ni de Pedro). El autor se centra también en las características sintagmáticas de ni... ni, que analiza como un sintagma conjuntivo en el que $n i_{2}$ es núcleo y ni $i_{1}$ es un índice morfológico de concordancia negativa (v. \$ 4.3 para más detalles). En cambio, Camacho (2003: 140) no observa asimetrías importantes entre $n i_{1}$ y $n i_{2}$, que interpreta como núcleos de sintagmas funcionales. En cambio, el autor ofrece un análisis diferente para $O_{1}$ $\mathrm{y}_{2}$, ya que considera que la primera partícula es un operador distributivo y la segunda, núcleo de un sintagma funcional (Camacho, 2003: 138)7. Por su parte, Aldaber (2018) retoma un análisis más clásico de estas partículas, que interpreta como núcleos de sendos sintagmas conjuntivos.

En suma, como se desprende de los párrafos anteriores, los trabajos sobre o... o y ni... ni se han centrado en el estudio de las posibles diferencias semánticas y distribucionales de estas partículas con las correspondientes simples o bien con aquellos elementos a los que parecen oponerse o por los que se pueden parafrasear. Los estudios acerca de las propiedades gramaticales de estas construcciones y de sus características fraseológicas internas son menos habituales y se enmarcan por lo general en la gramática generativa, con excepción de Conti (2018a). De estas aportaciones, además, solo Camacho (2003) y Aldaber (2018) tratan ni... ni y o... o, si bien con resultados interpretativos muy diferentes. En ninguno de los trabajos citados se relacionan las partículas $n i_{1}$ y $O_{1}$ con el foco, y tan solo en el estudio de Conti (2018a) se cuestiona la naturaleza coordinante de estas partículas ${ }^{8}$.

\section{Aspectos metodológicos: obtención y tratamiento de ejemplos}

Para la descripción de las estructuras objeto de estudio, en este artículo se ha partido del análisis de ejemplos extraídos del Corpus del Español del Siglo XXI de la RAE (CORPES XXI), en concreto, del subcorpus de textos escritos de España. Los ejemplos de cada estructura se han obtenido tras buscar las formas ni y o (sin establecer clase de palabra) con un intervalo de 10 a derecha e izquierda de ni y o, respectivamente. Este intervalo permite documentar tanto cláusulas como segmentos subclausales. A partir de los ejemplos (seleccionados por orden de aparición en las concordancias, ordenadas por año ascendente y sin criterio), se formaron sendas muestras de 100 ejemplos. Para obtener los 100 ejemplos de la estructura con 0... o, se revisaron un total de 2780 ocurrencias, de las que se descartaron aquellos casos (mayoritarios) en los que o y o estaban próximos, pero no formaban parte de una estructura correlativa, y aquellos otros en los que $o_{1}$ aparecía junto a bien. En el caso de ni... ni, se obtuvieron los 100 ejem-

7 En concreto, Camacho (2003: 38-40) considera que las conjunciones coordinantes no son núcleos de un sintagma conjuntivo, sino núcleos funcionales con contenido proposicional que copian los rasgos de otra categoría funcional de la oración.

8 En los demás trabajos se acepta implícita o explícitamente que ni... ni y o... o son coordinantes. 
plos tras revisar 400 ocurrencias, de las que se descartaron aquellos casos en los que ni aparecía como correlación de un término de polaridad distinto de ni, como en No estudia ni trabaja, y en los que ni era partícula focal fuera de estructura coordinante, como en No tengo ni idea.

En el fenómeno analizado en la sección 4.1.2, se amplió la búsqueda a un total de 339 casos para las secuencias no/ni... ni seguidas de sino. Se han realizado, asimismo, búsquedas específicas cuando se ha querido constatar las posibilidades combinatorias de $O_{1}$ y $n i_{1}$, como en la sección 4.1 para las combinaciones de / y $\mathrm{O}_{1} /$ e /y $n i_{1} /$ y, en la sección 4.3, para la combinación de o... o y ni... ni con preposiciones y posesivos.

El empleo de ejemplos procedentes de muestras reales de lengua insertas en un texto permite, por un lado, alejarse de las limitaciones de la propia competencia lingüística (más constreñida cuando se trata de ilustrar patrones de oraciones complejas o compuestas) y facilita, por otro, el estudio de los factores pragmáticos que puedan condicionar el uso de las estructuras aquí estudiadas. De hecho, el análisis de estas secuencias en un contexto discursivo pone de manifiesto la función pragmática de las partículas $\mathrm{O}_{1}$ y $n i_{1}$, a la que no se ha prestado especial atención en la bibliografía previa.

Cabe señalar, por último, que este artículo no se propone ofrecer un estudio de corpus ni estadístico, sino tan solo dejar constancia del funcionamiento observado en las muestras analizadas.

\section{Problemas del análisis de $\boldsymbol{O}_{1}$ y $n i_{1}$ como coordinadores}

\subsection{Combinación de $\boldsymbol{o}_{1}$ y $n i_{1}$ con la conjunción copulativa y}

A partir de la obra de Dik (1968), es lugar común en la bibliografía sobre coordinación señalar como prueba de reconocimiento de la naturaleza coordinante de una conjunción su incompatibilidad con otros nexos coordinantes, especialmente con la conjunción copulativa. Para el caso del español, Franchini (1986: 196) establece distintas pruebas combinatorias para el reconocimiento de lo que el autor denomina coordinadores, que opone a subordinadores y matizadores. En concreto, Franchini (1986: 196) señala que9:

a) Una partícula $x$ será un matizador si admite la estructura $M_{1}$ y $\times M_{2}$, donde $M$ se refiere a los miembros enlazados e y se corresponde con la conjunción copulativa no marcada (p. ej. Hace frío y, por eso, no salgo).

b) Una partícula $x$ será un subordinador si admite la estructura $M_{1} \times M_{2} y \times M_{3}$ (p. ej. No vendré porque llueve y porque hace frío).

9 Los ejemplos de los párrafos (a-c) son de Franchini (1986: 197). 
c) Una partícula $x$ será un coordinador si $x$ no admite ninguna de las estructuras anteriores (p. ej. *lápices rojos y y azules; lápices rojos y azules y y verdes).

Como se mostrará a continuación, las partículas objeto de estudio muestran distintos patrones ante estas pruebas, hecho que ya fue señalado por Franchini (1986: 200). En efecto, si bien $\mathrm{O}_{2}$ y $n i_{2}$, como cabía esperar, no admiten ninguna de las dos estructuras anteriores - lo que parece legitimar su análisis como coordinadores (p. ej. *o rojos y o azules; *o rojos o azules y o verdes) -, $\mathrm{O}_{1}$ y $n i_{1}$ pueden aparecer precedidos por y con total normalidad, como se muestra en los ejemplos (3) y $(4)^{10}$.

(3) a. Siempre hay huecos o sobrantes en lo que nos proponemos contar yo bien callamos lo que sobra, obien rellenamos las ausencias con invenciones o con hipótesis o con juicios.

b. i(...) no tenemos que comer a la fuerza la sopa, yo vamos desnudos o vamos sin ropa!

c. Evidentemente, esos hombres estaban bien entrenados, yo bien no acudieron a la cita por razones que desconozco o también utilizaron personas interpuestas para recoger la información.

d. Te cogen y o vas con ellos voluntario o te fusilan.

e. Lo pensé, qué decir, yo puedo seguirte el rollo y que creemos un vínculo más o menos afectivo para llevar mejor esto o soy honesta con lo que me transmitís y no entro a jugar a un juego.

(4) a. Si acaso me hizo recordar a mi madre, que no es intelectual y ni dirige seminarios ni da conferencias como la suya.

b. Me dio rabia que mamá se riera del miedo de Agustín y me dio rabia que me viera llorar y ni me abrazara ni me tocara.

c. No cumplía el horario convenido y ni fregaba ni barría.

d. La mujer lo toma como un halago, se echa a reír, se encoge de hombros, yniasiente ni niega.

Del mismo modo, las partículas $O_{1}$ y $n i_{1}$ pueden aparecer precedidas por la conjunción pero, como se ilustra en los siguientes ejemplos del CORPES XXI:

(5) a. Mientras tanto seguía intentando a toda costa conseguir entrevistas de trabajo, pero o bien no tenía suerte o no sabía cómo hacerlo.

b. Era evidente que quería hablar, pero o no sabía cómo o no sabía por dónde empezar.

c. Se lo supliqué en silencio, con la mirada, pero o no lo captó o no me hizo caso. 
d. Al parecer había tenido dos o tres, con uno de los cuales habíamos llegado a convivir un verano, pero o bien nunca los consideró con la suficiente seriedad, o bien yo era demasiado pequeño para otorgarles esa categoría.

(6) a. De buena gana habría llamado su atención y les habría hecho unas cuantas preguntas, pero ni la prudencia lo aconsejaba ni la buena marcha de la peluquería me permitía seguir ausente de ella.

b. Me habría fumado un cigarro, pero ni tenía cigarros ni quería que mi boca le supiera a humo.

c. Le estuve esperando durante el resto de la semana por si se le ocurría ir a las horas en las que ya sabía que trabajaba, pero ni atravesó la puerta ni un día le descubrí mirando con fascinación la vieja foto del puente sobre el río Liffey.

d. Lo cierto es que Dick Dalton fue asaltado, acariciado, mordido, pero ni amó ni fue amado.

En opinión de Franchini (1986: 200), ejemplos como los de (3) y (4) no invalidan el tratamiento de $\mathrm{O}_{1}$ y $n i_{1}$ como coordinadores, ya que considera que estos elementos forman parte de un coordinador discontinuo al que no es aplicable la estructura $M_{1} y \times M_{2}$. En concreto, argumenta que la estructura implicada en estos casos es siempre $M_{1} y M_{2}$, con la particularidad de que $M_{2}$ está compuesto por coordinación mediante un coordinador discontinuo, tal y como se recoge en (7):

(7) (Franchini 1986: 200)

$$
\left[M_{1} y[\underbrace{\left.x A_{1} \times A_{2}\right]}_{M_{2}}]\right.
$$

En opinión del autor, por tanto, en ejemplos como [...] yo vas con ellos voluntario o te fusilan, la secuencia o vas con ellos voluntario o te fusilan es $\mathrm{M}_{2}$ (o segundo coordinando de $y$ ), de modo que la adyacencia de [...] y o [...] estaría reflejando dos procesos de coordinación distintos, totalmente compatibles.

Sin embargo, como se mostrará en las secciones 4.2-4.3, $O_{1}$ y $n i_{1}$ presentan además otras características distribucionales que invitan a tratar estas partículas fuera de la clase de los coordinadores.

\subsection{Posición de $o_{1}$ y $n i_{1}$}

$\mathrm{O}_{1}$ y $n i_{1}$ muestran propiedades distribucionales distintas de los coordinadores copulativos y disyuntivos. En primer lugar, $\mathrm{O}_{1}$ y $n i_{1}$ preceden inmediatamente al primer elemento unido por $\mathrm{O}_{2} / \mathrm{ni}_{2}$, como se observa en los siguientes ejemplos ${ }^{11}$ :

11 Ejemplos extraídos del CORPES XXI. 
(8) a. Tan quieta que no se movían [ni las hojas desplegadas del periódico ni la falda por media pantorrilla que llevaba].

b. No puedes irte [ni hoy ni mañana].

c. No se sentía [ni capaz ni con fuerza de hacerlo].

d. Porque [ni les había chocado la llegada de un señor al restaurante, ni sabían quién era Tere, ni, mucho menos, a lo que se dedicaba].

(9) a. Bastante tuve con la tía Eduvigis... [iO casada o al convento!]

b. Me colocó entre la espada y la pared: [o él o mi padre].

c. Me ha puesto un ultimátum. [O todo o nada]. [O irnos a vivir juntos o la fiesta se ha acabado].

d. Se lo supliqué en silencio, con la mirada, pero [o no lo captó o no me hizo caso].

A esto se suma que, a diferencia de los coordinadores copulativos y disyuntivos del español, que son recursivos, $\mathrm{O}_{1}$ y $n i_{1}$ solo pueden aparecer una vez, ya que están ligados al primer elemento del conjunto. En cambio, $\mathrm{O}_{2}$ y $n i_{2}$, como se aprecia en los ejemplos del CORPES XXI Ese hombre, $\underline{\underline{O}}_{\underline{1}}$ tiende a rechazar su masculinidad, $\underline{\underline{O}}_{2}$ miente $\underline{\underline{O}}_{2}$ no dice la verdad y No hay $\underline{n i_{1}}$ moreras $\underline{n i_{2}}$ lechugas $\underline{n i_{2}}$ ningún tipo de vegetación que tú conozcas, pueden repetirse para unir dos o más segmentos.

Una prueba adicional de que $o_{1}$ y $n i_{1}$ no son coordinadores es su naturaleza facultativa. En concreto, $O_{1}$ siempre es prescindible (p. ej. La gente normal, toda la gente, [o] se muere de algo malo, o se muere de repente]) y ni, solo es obligatorio cuando no está bajo el alcance de una negación (p. ej. [*Ni] puedo correr ni puedo hacer movimientos bruscos frente a No puedes irte [ni] hoy ni mañana) (v. § 4.3 para más detalles).

En resumen, la posición siempre antepuesta al primer miembro del conjunto, la imposibilidad de recursión y la naturaleza facultativa de $o_{1}$ y de $n i_{1}$ (este último, en los contextos en los que no se incumplen las restricciones de polaridad negativa) parecen indicar que se trata de partículas muy condicionadas semántica y pragmáticamente cuyo cometido, además, no es coordinar o unir elementos. Como se detallará en el apartado 5.1, $O_{1}$ y $n i_{1}$ indican en concreto que los miembros unidos tienen un estatus semántico y pragmático especial.

\subsection{Restricciones combinatorias de $0 . . .0$ y ni... ni}

O... o y ni... ni presentan restricciones distribucionales y combinatorias que las alejan de las construcciones con coordinadores simples (en el primer caso, de la disyuntiva o y, en el segundo, de la copulativa $y$ ).

Como observó Bosque (1994), ni no puede coordinar términos de una preposición (v. las oposiciones entre 10a y 10b y entre 11a y 11b) (Bosque, 1994: 188 y 193), ni subordinadas dentro 
de completivas con que (v. 12a y 13a frente a 12b y 13b) (Bosque, 1994: 189 y 193). Asimismo, el autor señala que, entre no y ni o entre ni y ni no puede haber un posesivo (Bosque, 1994: 188 y 193) (v. 14-15) 12.

(10) a. *No he visto retratos de [Juan ni Pedro].

b. No he visto retratos [de Juan ni de Pedro].

(11) a. *Con [ni Juan ni Pedro]

b. [Ni con Juan ni con Pedro]

(12) a. *No pedí que [Juan se fuera ni Luis se quedara].

b. No pedí [que Juan se fuera ni que Luis se quedara].

(13) a. *No pedí que [ni Juan se fuera ni Luis se quedara].

b. No pedí [ni que Juan se fuera ni que Luis se quedara].

(14) No he visto (*tus) retratos de Juan ni de Pedro.

(15) No vi (*tus) fotos ni de Juan ni de Pedro.

De acuerdo con los datos que ofrece CORPES XXI, parece que, en efecto, ni no permite la irrupción de posesivos dentro de frases nominales ni es aceptable con términos de preposición, salvo en el siguiente caso puntual (v. 16):

(16) Después de un periodo de ni guerra ni paz, en el que se confió a la Autoridad Nacional Palestina la tarea de mantener un orden precario en sus guetos (...).

En cambio, la presencia de ni... ni dentro de subordinadas completivas que dependen de un verbo subordinante negado está sujeta a cierta variación, pues parece aceptable en ejemplos como los siguientes:

12 Estas restricciones de combinatoria son prueba, en opinión de Bosque (1994), de que el sintagma conjuntivo proyectado por niz es un término de polaridad negativa que se mueve en Forma Lógica al especificador del sintagma de negación de no. Al moverse, dicho sintagma deja una huella que debe ser debidamente interpretada. La imposibilidad de que ni una términos de preposición, como en los ejemplos (10a) y (11a), se explica, de acuerdo con el autor, por el hecho de que las preposiciones son rectores débiles en español que no pueden identificar la huella dejada por el sintagma conjuntivo al moverse en Forma Lógica (Bosque, 1994: 188). En lo que se refiere a los ejemplos de (12a) y (13a), la explicación radica de nuevo en la imposibilidad de interpretar la huella dejada por el nudo hermano del complementante que cuando se mueve (Bosque, 1994: 189). Por último, en los casos de (14) y (15), el posesivo interrumpe la relación entre no y la frase de polaridad negativa. La explicación de Bosque (1994) no sería extrapolable a estas mismas restricciones observadas para o... o que se muestran más adelante, ya que en este caso no hay ningún término de polaridad negativa ni cabe interpretar que estas restricciones se deban a movimientos del sintagma negación. 
(17) No quiere que ni estudie ni trabaje (Jiménez Juliá, 1995: 77).

(18) a. Estos estudios no concluyen que ni limpie el organismo de toxinas ni ayude a mantenerse en forma.

b. No había considerado que ni él diera más de sí ni a ella le interesara el mundo de la fotografía.

c. Parece que no interesaba que niel campesino cultivase la trufa ni el científico la estudiase.

Para la secuencia o... o no se han señalado en la bibliografía previa restricciones de combinatoria como las que indica Bosque (1994) para NEG/ni... ni. Cabe mencionar, sin embargo, que también o... o es poco aceptable con términos de preposición (v. 19a frente a 19b) y no parece ser compatible con complementos de nombres con posesivos (v. 20):

(19) a. Es un trabajo de *[o Juan o Pedro].

b. Es un trabajo de [Juan o Pedro].

(20) Vio (*tus) fotos o de Juan o de Pedro.

O... o es, en cambio, completamente natural con cláusulas introducidas por conjunciones subordinantes (v. 21), dado que no es una estructura ligada a la presencia de una partícula negativa:

(21) Pero verás que o nos traslada o nos echa.

El hecho de que ni... ni y o... o muestren las mismas restricciones de combinatoria (con términos de preposición y con posesivos) parece poner de manifiesto que ambas secuencias comparten pautas gramaticales ligadas a su naturaleza correlativa y, más particularmente, a la naturaleza correlativa de $O_{1}$ y $n i_{1}$, como se intentará demostrar en la sección 5 . En cambio, las restricciones de ni en subordinadas completivas parecen estar relacionadas con su naturaleza como término de polaridad negativa, ya que se trata de un rasgo singular de esta estructura que no presenta $0 . . .0$.

\section{Propuesta alternativa de análisis: $\boldsymbol{O}_{1}$ y $n i_{1}$ como partículas correlativas de naturaleza focal}

Las partículas $\mathrm{O}_{1}$ y $n i_{1}$ del español se alejan de las propiedades atribuidas a los coordinadores, pero se aproximan, como se mostrará en esta sección, a las pautas de comportamiento de los correlativos, una macroclase de palabras que comparten propiedades gramaticales y tienen una acusada función pragmática en las lenguas.

Los correlativos se caracterizan en conjunto por inducir o abrir una relación formal y semántica de dependencia entre el segmento sobre el que tienen alcance y un segundo segmento, 
que contiene la partícula que marca el correlato (p. ej. Llévate tantos $\underline{\underline{1}}_{1}$ libros cuantos $\underline{\underline{2}}_{\underline{2}}$ necesites) (Conti, 2018b) ${ }^{13}$. Los correlativos y las partículas del correlato son normalmente formantes sin contenido léxico o altamente gramaticalizados que pertenecen a distintas clases de palabras, como pronombres, determinantes o cuantificadores (p. ej. Llévate tantos libros $_{1}$

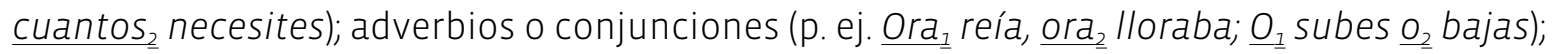
o una combinación de estas palabras (p. ej. Cuantos más libros leo, más ganas tengo de leer). En ocasiones, los correlativos son partículas de difícil adscripción categorial, principalmente cuando son resultado del doblado formal de palabras no léxicas, como sucede con $o_{1}$ y $n i_{1}$.

En lo que se refiere a las características gramaticales, los correlativos exigen (o presuponen en las estructuras truncas) un correlato formal (p. ej. $\mathrm{O}_{2}$ y $n i_{2}$ en el caso que nos ocupa), a lo que cabe añadir que no admiten la recursión (por ejemplo, no permiten más de un correlativo y, a menudo, tampoco más de un correlato) y muestran ciertas restricciones de posición (p. ej. respecto del correlato, al que siempre preceden).

Los correlativos y sus correlatos aparecen tanto en estructuras de subordinación (p. ej. Llovió tanto que se inundaron las calles) como de coordinación (p. ej. Tanto Pedro como Luis acudieron al evento) y establecen entre sí distintas relaciones configuracionales, según la construcción: pueden formar parte de un mismo constituyente, como sucede en tanto Pedro como Luis, o ser constituyentes distintos, como sucede en Trajo tantos regalos a su hijo que no tenían dónde guardarlos.

Se ha señalado, asimismo, que las construcciones correlativas son expresiones maximalizadoras (Lipták, 2009: 8-10), bien por referir a un individuo definid o y específico o a todos los individuos de una clase, como sucede con los correlativos relativos del hindi (p. ej.jo... us... 'que... esa'); bien por expresar grado máximo, como sucede con las expresiones escalares de las comparativas correlativas del inglés ( $p$. ej. The more you read, the less you understand) o con las consecutivas ponderativas del español (p. ej. Llovió tanto esos días que se inundaron las calles) (Conti, 2018b). Como se mostrará en este artículo, los correlativos $O_{1}$ y $n i_{1}$ se pueden sumar también a esta tendencia general, pues son partículas que, al expresar exclusión (solo una alternativa del conjunto; ninguna alternativa del conjunto), se pueden relacionar con significados maximalizadores.

Además, los correlativos desempeñan una acusada función pragmática en las lenguas, ya que su aparición determina la estructura informativa de la oración: en unos casos se pueden

13 Las construcciones correlativas presentan una considerable variación interna (p. ej. pueden ser comparativas, ponderativas, relativas, etc.) y se documentan en un amplio abanico de lenguas. Sin embargo, se conoce poco del comportamiento de estas construcciones, estudiadas con cierto detalle en lenguas como el hindi, el inglés, el neerlandés y el noruego (v. el volumen de Lipták, 2009). No se ha ofrecido, sin embargo, un estudio de conjunto sobre las correlaciones en otras lenguas de larga tradición gramatical, como el español. 
emplear para señalar el tópico, entendido como aquello sobre lo que se habla y que es importante en el discurso (v. Lipták, 2009: 12-13), y, en otros, para señalar el foco (Conti, 2018b). En lo que se refiere a las construcciones correlativas disyuntivas y copulativas, se ha observado que las formas inglesas either, both y neither presentan características de posición, alcance y prosodia similares a las de las partículas focales only, even, etc. (Hendriks, 2001, 2003 y 2004; Johannessen, 2005), lo que ofrece una vía de estudio alternativa a la interpretación de estas partículas como coordinadores ${ }^{14}$.

En las siguientes secciones, se repasarán esas características y se contrastarán con los patrones observados para $O_{1}$ y $n i_{1}$.

\subsection{Partículas focales y propiedades semánticas, pragmáticas y prosódicas de $0 . .$. o y ni... ni}

Las partículas $\mathrm{O}_{1}$ y $n i_{1}$ comparten varias propiedades con las partículas focales. Como estas, son palabras gramaticales con poco peso fónico que pertenecen a una serie cerrada y cuya aparición se relaciona con la marcación del foco ${ }^{15}$, entendido este como aquel segmento de la oración con mayor peso informativo o bien con carácter enfático o contrastivo.

Desde el punto de vista semántico, $O_{1}$ y $n i_{1}$ encajan dentro de las que König (1991: 33) denomina partículas focales excluyentes o restrictivas, ya que marcan la elección de un elemento sobre una serie de alternativas que se excluyen. Muestran, además, efectos semánticos atribuidos a las partículas focales (König, 1991: 33) -como el comprender semánticamente la correspondiente oración sin partícula (p. ej. No trajo ni pan ni vino / No trajo pan ni vino) - y dependen del contexto discursivo (König, 1991: 4-5), pues su aparición refleja un posicionamiento del emisor ante el contexto discursivo, como se detallará de inmediato en las siguientes secciones.

\subsubsection{Propiedades de $0 . . .0$}

O... o, como se ha señalado en la bibliografía previa (v. § I), expresa disyunción exclusiva, dado que supone la elección de uno de los miembros del conjunto y excluye a los restantes. Cabe añadir a este respecto que la presencia de $O_{1}$ puede expresar preferencia por parte del emisor hacia el primer elemento de la correlación. En tales casos, $o_{1}$ cumple la función de señalar que el primer miembro de la disyunción es el preferido por el emisor de entre los demás miembros de la estructura, que se entienden como alternativas disponibles, pero excluidas. La partícula $\mathrm{O}_{1}$ puede focalizar, pues, el miembro sobre el que tiene alcance inmediato, al que dota de una mayor relevancia pragmática o contraste. Dicha función se observa especialmente en tres

14 Para otros análisis de estas construcciones del inglés, véanse Larson (1985) y Schwarz (1999).

15 Véanse König (1991: 14) y Sudhoff (2010: 16) para esta definición de partícula focal. 
tipos de ejemplos con o... o: aquellos en los que lo expresado por el correlato tiene consecuencias adversas y, por tanto, cabe interpretar que el emisor lo considera el menos deseable de la serie (v. 22); aquellos en los que se omite el correlato y se sobreentiende, en consecuencia, que la información omitida es también adversa (v. 23); y, finalmente, aquellos en los que el correlato es un enunciado inespecífico, con una fuerza ilocutiva diferente del primer elemento, que viene a cerrar la estructura correlativa, pero que realmente no ofrece una alternativa que excluir en la correlación disyuntiva (v. 24)

(22) a. O te dejas televisar o no toreas.

b. O se salvan juntas, o juntas se despeñan.

c. Te lo repito, Juana: o te regeneras o todo habrá acabado definitivamente entre nosotros.

d. O Tere cambiaba de vida o... la dejaba, con todo el dolor de su corazón, pero la dejaba.

e. O encuentras los papeles o esto va a acabar aún peor de lo que está.

f. Pero en esto a lo que nos dedicamos, o espabilas o te espabilan, ¿no?

g. Siempre digo lo mismo... o tienes un BMW o tienes un coche.

(23) a. O te regeneras $0 . .$.

b. O te vienes a la cama ahora mismo o te...

(24) a. O vivimos juntos y con todas las consecuencias o nunca más.

b. Uno gimoteaba y se mordía la lengua de dolor o para no chillar o qué sé yo para qué.

Cabe señalar, para cerrar este apartado, que la partícula $O_{1}$ abre una curva tonal de anticadencia o semianticadencia que se cierra con la aparición de la partícula del correlato $\left(\mathrm{O}_{2}\right)$. Este tipo de rasgo prosódico se asocia en la bibliografía especializada con la presencia de un foco contrastivo, lo que parece apuntar a que el primer segmento al que precede $o_{1}$ tiene un estatus pragmático especial, en oposición al resto de los miembros del conjunto ${ }^{17}$. Esto se observa relativamente bien para el caso de $O_{1}$, que, como se mostró unas líneas más arriba, marca a menudo la preferencia del emisor por el primer elemento del conjunto, sobre el que tiene alcance inmediato (foco contrastivo), y excluye consiguientemente los demás miembros.

\subsubsection{Propiedades de ni... ni}

El análisis semántico y pragmático de $n i_{1}$ plantea problemas diferentes según se encuentre o no bajo el alcance de una partícula negativa (p. ej. no). Este doble comportamiento opone $n i_{1}$

16 Los ejemplos (22)-(24) se han extraído del CORPES XXI.

17 Véase Féry (2013) para la noción de prominencia prosódica asociada al foco contrastivo. Como se mostrará en la sección 5.3, Hendriks (2001: 2) observa que la partícula either '0...' del inglés debe tener alcance sobre un foco contrastivo dentro del primer conjunto, lo que parece suceder al menos en el caso de $O_{1}$. 
a $n i_{2}$, ya que esta última es siempre término de polaridad negativa, pues su aparición solo es posible si se encuentra bajo el alcance de una partícula negativa (p. ej. Trabaja *ni estudia)².

Así pues, se pueden diferenciar dos pautas para ni $i_{1}$ : cuando está bajo el alcance de una partícula negativa, $n i_{1}$ es facultativo desde el punto de vista gramatical, ya que su presencia no legitima $n i_{2}$ (p. ej. No trajo [ni] ajos ni cebollas); cuando $n i_{1}$ es ella misma una partícula negativa, su presencia es obligatoria para legitimar ni, (p. ej. *[Ni] estudia ni trabaja). En el primer caso, $n i_{1}$ no tiene la función de negar el miembro del conjunto al que precede ni tampoco posee la función de legitimar $n i_{2}$, ya que de ambas cosas se ocupa la partícula negativa no o similares, como nunca (p. ej. Nunca visitó [ni] Madrid ni Berlín). En cambio, ni. puede ser una partícula negativa con capacidad para negar al segmento al que precede y legitimar $n i_{2}$ en ejemplos como Ni estudia ni trabaja, Ni Juan ni Pedro vinieron y Ni a Pedro ni a Luis les dieron al final la beca. En términos de análisis lineal de constituyentes, $n i_{\Perp}$, en cuanto partícula negativa, ocupa la primera posición del enunciado, bien por tener alcance sobre cláusulas (p. ej. Ni estudia ni trabaja), bien por tener alcance sobre partes de un sujeto preverbal (p. ej. Ni Juan ni Pedro vinieron) o sobre partes de un constituyente antepuesto (p. ej. Ni a Pedro ni a Luis les dieron al final la beca). Asimismo, se emplea en aquellos casos en los que se elide el material no focal del predicado (p. ej. Mira, "María Coraje” hace años que no la canto, ni aquí ni en ningún otro sitio) ${ }^{19}$.

Hecha esta sinopsis, cabe plantearse a continuación qué propiedades semánticas y pragmáticas tiene $n i_{1}$. Para ello parece adecuado centrarse en el análisis de aquellos contextos sintácticos en los que dicha partícula es facultativa (p. ej. No compré [ni] ajos ni cebollas). Una de las funciones pragmáticas de $n i_{1}$ en estos casos es la de orientar el alcance de la partícula negativa no hacia el segmento al que antecede. Es, por tanto, una partícula claramente focalizadora que permite la interpretación en línea del alcance de la negación, ya que centra el interés informativo sobre su segmento. Por ejemplo, sin la aparición de $n i_{2}$, el alcance de la negación en un ejemplo como (25) no se procesa hasta la aparición de ni; es decir, en el procesamiento en línea de (25), no puede estar negando a la feria o a su hermana, hasta el momento en el que se procesa ni, cuyo coordinando determina finalmente sobre qué elemento tiene alcance no. La aparición de $\mathrm{ni}_{1}$, como se muestra en (26), permite determinar mucho antes el alcance del operador negativo, lo que adelanta y facilita el procesamiento de la oración:

(25) Juan no fue a la feria con su hermana ni con su madre.

(26) Juan no fue a la feria ni con su hermana ni con su madre.

Además de esta cualidad, ligada al alcance de la negación y al procesamiento en línea de las oraciones, el análisis de los datos obtenidos en este trabajo permite constatar que ni... ni ex-

18 Esto no ocurría, sin embargo, en castellano medieval, estadio de la lengua en el que $n_{2}$ era todavía partícula negativa.

19 Este último ejemplo procede del CORPES XXI. 
presa un conjunto de elementos que, de acuerdo con el emisor, son esperables o conocidos en el discurso o en el contexto y cuya negación es, en consecuencia, prominente. Así, en la oración citada antes de No compré ni ajos ni cebollas, cabe suponer que los ajos y las cebollas eran relevantes en la conversación, de ahí que la negación subraye su relevancia discursiva.

Además, los elementos de la correlación de ni... ni se presentan a menudo como partes de un conjunto cerrado, sin alternativas posibles o de interés, como se ilustra en (27):

(27) Parece que no interesaba ni al campesino ni al científico andaluz.

En (27), en efecto, el empleo de ni... ni excluye la interpretación de que haya otros potenciales interesados en el cultivo de la trufa, aparte del campesino o del científico andaluz.

Sin embargo, no... ni carece de estas particularidades pragmáticas y permite con normalidad interpretar que hay otras alternativas a los miembros negados del conjunto, como se observa en (28), donde se excluyen los huevos de gallina, pava y pintada para aludir realmente a otros huevos, que constituyen alternativas necesarias a los miembros negados ${ }^{20}$.

(28) Los huevos que no sean de gallina, de pava ni de pintada se manipularán y transformarán separadamente, procediendo a su limpieza y desinfección posterior.

De hecho, de los 339 casos obtenidos del CORPES XXI en los que aparecen no/ni... ni con referencia explícita a una o varias alternativas introducidas por la adversativa sino, solo 18 son de ni... ni; el resto lo constituyen construcciones con no... ni..., sino, como la mostrada en (29)21.

(29) Y cuando volvió, no era el de antes ni el de después, sino un Andrés distinto.

De los 18 ejemplos mencionados, ni... ni se combina en la mayoría de los casos con propiedades (en función atributiva o modificadora) que o bien se consideran esperables o son conocidas (y a las que sino introduce una alternativa opuesta o que rompe con las expectativas del emisor; v. 30), o bien conforman una escala de opuestos con la que se relaciona la propiedad o propiedades alternativas expresadas por sino (v. 31):

(30) a. Debías tener un poco más de respeto por Anselmo, que no es ni un niño ni un tonto, sino un hombre digno y sabio.

b. Ahora no resulta ya ni chocante ni cómica, sino trivial y casi incomprensible.

c. Contra la luna, que ya no parece ni cruel ni burlona, sino alcahueta, vieja y acaso cobarde.

20 El ejemplo (28) es del CORPES XXI.

21 Los criterios de búsqueda han sido los siguientes: subcorpus de España, ficción, escrito; proximidad de ni a sino con un intervalo de 8 a la derecha. 
(31) a. La verdad es que, bien pensado, esa media sonrisa taimada, ni oblicua ni abierta sino todo lo contrario.

b. 0 al menos eso es lo que le pasa a Tomás, que no es ni un pessimist ni un optimist, sino lo uno y lo otro alternativamente.

c. (...) larga y espléndida cabellera ni rubia ni morena, ni lisa ni rizada, sino de un castaño en el que se entreveraban numerosas hebras doradas.

Sino también puede introducir una alternativa a entidades conocidas o esperables marcadas con ni... ni..., como se ilustra en (32):

(32) Lo malo es que los acompañantes del profesor no fueron ni artistas ni clientes de galerías, sino unos cuantos alumnos de sus cursos.

En lo que se refiere a las propiedades prosódicas, la partícula $n i_{1}$, al igual que $O_{1}$, abre una curva tonal de anticadencia o semianticadencia que parece cerrarse con la aparición del correlato $\left(n i_{2}\right)$. De nuevo, este rasgo prosódico está ligado a la función pragmática de $n i_{1}$, que focaliza su constituyente. Es decir, ni $i_{1}$ niega materia focal en una estructura informativa en la que no todo ha de ser informativamente relevante.

\subsection{Restricciones distribucionales de $o_{1}$ y $n i_{1}$ que las aproximan a las partí- culas focales}

La secuencia o... o presenta restricciones de combinatoria distintas de las mostradas por o, pero similares, en cambio, a las que se han observado para el inglés either y el neerlandés of (Hendriks, 2003: 4-5). Como se ilustra mediante los contrastes de (33) y (34), o puede coordinar elementos inferiores al sintagma, como profesor o investigador en (33a), viejo o estropeado en (33b) y comido o bebido en (33c), pero no así o... o, que no es aceptable en estos mismos entornos sintácticos (34). Parece, pues, que o... o, como sucede con either (Hendriks, 2003), solo combina sintagmas o proyecciones máximas (v. 35):

(33) a. Un buen [profesor o investigador]

b. Muy [viejo o estropeado]

c. Ha [comido o bebido] algo.

(34) a. *Un buen o profesor o investigador

b. *Muy o viejo o estropeado

c. ?Ha o comido o bebido algo.

(35) a. O un buen profesor o un buen investigador

b. O muy viejo o muy estropeado

c. O ha comido o (ha) bebido algo. 
Estas mismas restricciones se observan para ni... ni (v. 36) y ni (v. 37), aunque de manera más extrema para la marcación doble. En cambio, la conjunción y no está sujeta a estas condiciones (v. 38):

(36) a. *No necesitan un buen ni profesor ni investigador/No necesitan ni un buen profesor ni un buen investigador.

b. *No es un libro muy ni viejo ni estropeado / No es un libro ni muy viejo ni muy estropeado.

c. *No ha ni comido ni bebido nada / Ni ha comido ni (ha) bebido nada.

(37) a.?No necesitan un buen profesor ni investigador.

b. ?No es un libro muy viejo ni estropeado.

c. No ha comido ni bebido nada.

(38) a. Un buen [profesor e investigador]

b. Muy [viejo y estropeado]

c. He [comido y bebido] algo.

En suma, el hecho de que ni... ni y o... o solo combinen proyecciones máximas parece poner de manifiesto que $o_{1}$ y $n i_{1}$ condicionan el tipo de relación formal entre los miembros del conjunto. De nuevo, su comportamiento se aproxima más, como se ha mostrado en esta sección y en las anteriores, al de las partículas correlativas focales de otras lenguas.

\subsection{Posición de $o_{1}$ y $n i_{1}$ y estructura informativa}

Las partículas $\mathrm{O}_{1}$ y $n i_{1}$ siempre preceden inmediatamente a su dominio, a diferencia de las partículas focales, que muestran una mayor movilidad dentro de la oración. En este punto, por tanto, se alejan de las partículas inglesas either y neither, que, de acuerdo con Hendriks (2001), pueden suceder o preceder a su dominio y no exigen su adyacencia. En concreto, Hendriks (2001: 2) señala que either, a diferencia de la conjunción or, puede aparecer antepuesto (como en Jane either ate [SD rice] or [SD beans]) y también puede posponerse al elemento sobre el que tiene alcance, como en Jane either ate rice or she ate beans, donde either domina la oración en la que está inserto, y no solo el segmento que aparece detrás de la partícula. Además, y siempre según Hendriks (2001: 2), either debe tener alcance sobre un foco contrastivo dentro del primer conjunto, de ahí la aceptabilidad de Jane ate either rice or cereals, pero no de *Jane ate either rice or she didn't (frente a Jane ate rice or she didn't) ${ }^{22}$.

22 Hendriks (2001) concluye que either es una partícula focal, posiblemente de naturaleza adverbial (no conjuntiva), que se genera en el léxico junto a la conjunción or, con la que forma un mismo núcleo. En opinión de la autora, either puede moverse según los requisitos de alcance y dominio propios de las partículas focales. 
En lo que respecta a $O_{1}$, cabe señalar en primer lugar que se trata de una partícula correlativa focal cuyo dominio más documentado en los ejemplos es la cláusula, a la que siempre precede ${ }^{23}$. La naturaleza focal de la cláusula dominada por $O_{1}$ es poco compatible con la expresión de información compartida o conocida, es decir, con la aparición del tópico, de ahí que estas cláusulas carezcan a menudo de sujeto explícito (v. 3924:

(39) a. O te dejas televisar o no toreas.

b. O se salvan juntas, o juntas se despeñan.

c. Es que aquí o no llegamos o nos pasamos.

d. En cuanto a ustedes dos, o consumen o prosiguen el galanteo en un meublé.

Cuando el sujeto se explicita dentro de la cláusula, se ha de entender que este forma parte del foco. En estos casos, son frecuentes los sujetos contrastivos, a menudo pronominales (v. 40):

(40) a. Con que o se casa usted con ella como Dios manda o le pego una perdigonada de sal [...].

b. O me muero yo, o se tiene que morir alguna de ellas.

c. O tú deliras o ese tío es imbécil.

d. O el etarra era muy bueno o él perdía reflejos.

e. O el mundo era incomprensible per se, o yo no partía de los mismos supuestos que los demás.

Asimismo, caracteriza a estas estructuras correlativas el poder codificar el tópico mediante la anteposición del sujeto a la estructura con o... o. En este caso, el sujeto se comporta a efectos pragmáticos, prosódicos y estructurales como un tópico descolgado, pues expresa información compartida y ocupa una posición externa, marcada por la pausa fónica que lo sigue (v. 4I):

(41) a. La gente normal, toda la gente, [o se muere de algo malo, o se muere de repente].

b. El catalejo ése [o viene desenfocado de fábrica o es de pego].

c. Ese hombre, [o tiende a rechazar su masculinidad, o miente o no dice la verdad].

d. En el fondo los compañeros [o le daban la razón o no se atrevían a quitársela].

23 De acuerdo con los datos obtenidos en el CORPES XXI, se observan ciertas preferencias de uso en el tipo de constituyente combinado mediante o... o y ni... ni. O... o se emplea sobre todo con cláusulas y, en menor medida, con otra clase de constituyentes inferiores, como se observa en el ejemplo del CORPES XXI Los domingos en mi pueblo había dos opciones: o al rosario con tu abuela o al fútbol con tu padre (en concreto, se trata de 16 casos de los 100 recopilados). 
Por su parte, la partícula $n i_{1}$ es un focalizador con un dominio más amplio que $O_{1}$ desde el punto de vista categorial, como se ilustra en (42)-(44) (con frases nominales en 42, con frases adverbiales y preposicionales en 43 y con adjetivo en 44$)^{25}$.

(42) a. No se movían ni las hojas desplegadas del periódico ni la falda por media pantorrilla que llevaba.

b. Laura, la que escuchaba sin interrumpir ni la palabra ajena ni los telediarios.

(43) a. No puedes irte ni hoy ni mañana.

b. No estaba esa mañana ni para aguantar penas ajenas ni para hablar del tiempo.

(44) Porque no se sentía ni capaz ni con fuerza de hacerlo.

Cuando su dominio es la cláusula, $n i_{1}$ muestra las mismas pautas que $o_{1}$ en lo que se refiere al sujeto, que se omite en numerosos ejemplos (v. 45). Cuando se explicita dentro de la estructura correlativa, el sujeto es focal y suele ser enfático o contrastivo (v. 46):

(45) a. Ni creo que este tipo sea peligroso ni, en todo caso, sucedería lo más mínimo.

b. Ni conocen esto ni conocen a su gente de aquí.

c. Él sería un caos, que lo era, un tipo salvo para sus letras sin horario fijo, que ni lo tenía ni quería tenerlo.

d. Fue tan gratuito como parece. Y yo fui tan cobarde como parece. Ni le partí la cara ni me acordé de su familia.

(46) a. No le entró a José Antonio Cambronero la risa porque ni estaba la mañana para risas ni aquél era el lugar más idóneo para hacer risas.

b. Porque ni les había chocado la llegada de un señor al restaurante, ni sabían quién era Tere, ni, mucho menos, a lo que se dedicaba.

c. Porque ni ese coche era para mujeres ni a él lo afectaba el alcohol ni le daba miedo la guardia civil.

d. Pero ni yo estaba de humor ni su abogado podía impresionarse con él.

e. Lo tuteó y desempolvó sin querer una voz suplicante, aunque ni Jesús Romero estaba para distinguir matices ni Antonio Carreño de suerte.

Al igual que sucede con o... o, ni... ni permite sujetos topicales antepuestos a la estructura correlativa (v. 47):

25 Los ejemplos son del CORPES XXI. El amplio espectro de $n i_{1}$ podría explicar su extensión como partícula focal independiente, fuera de la estructura correlativa (p. ej. No quiero ni verlo). 
(47) a. Aunque Lalo [ni sabía dónde trabajaba ni nada más de ella].

b. Y no se le iba de la mente que su marido [ni aparecería por allí en ningún caso ni por casa hasta las tantas].

c. Eso [ni contaba ni lo iba a contar].

En suma, la aparición de $O_{1}$ y $n i_{1}$ condiciona la estructura informativa de la oración, principalmente, en lo que afecta a la codificación del sujeto topical. Esto apunta, de nuevo, a la estrecha relación de $O_{1}$ y $n i_{1}$ con la marcación del foco y, por extensión, con las partículas correlativas focales de otras lenguas.

\section{6. $O_{1}, n i_{1}$ y estructura de constituyentes}

Los fenómenos descritos en este artículo parecen apuntar a que $o_{1}$ y $n i_{1}$ no son conjunciones, no unen segmentos y tienen una clara función pragmática, relacionada con la señalización del foco. Resulta poco plausible, por tanto, que estas partículas se puedan proyectar en solitario como núcleos de un sintagma conjuntivo ${ }^{26}$ o que sean marcadores de enlace que unen segmentos, si se adopta una explicación funcional del fenómeno.

El análisis de $O_{1}$ y $n i_{1}$ como operadores es probablemente la interpretación más adecuada para estas partículas. Esta hipótesis se ve respaldada por el hecho de que $o_{1}$ y $n i_{1}$ son partículas enfáticas que repercuten sobre la fuerza ilocutiva de la oración (no aceptan modalidades distintas de la declarativa, p. ej. *O ven o vete) y sobre otros operadores de alcance oracional, como la negación en el caso específico de $n i_{1}$. El modo de representar estos operadores en la estructura de constituyentes o bien en la representación estructural de la oración dependerá, en buena lógica, del modelo teórico adoptado; por ejemplo, como núcleo de sintagma funcional (sintagma foco, sintagma correlativo, etc.) o como palabra que ocupa un hueco estructural proyectado por otros núcleos; o bien como marca operacional que no interviene en la formación de frases, si se adopta un enfoque funcional. Esta cuestión excede los límites del presente artículo, pero no invalida, creemos, la hipótesis general aquí defendida.

\section{Conclusiones}

En este artículo se ha intentado demostrar que la primera partícula de las secuencias o... o y ni... ni, que se ha analizado tradicionalmente como coordinador o parte de un coordinador

26 Si se aplica una teoría configuracional endocéntrica, sería más plausible postular, como hace Hendriks (2001) para either, que $O_{1}$ y $n i_{1}$ son partículas focales que se generan en el léxico junto a sus respectivas conjunciones, con las que forman un mismo núcleo, y que, al igual que either, se mueven para tener alcance sobre el segmento focalizado. 
discontinuo, muestra paralelismos con las partículas correlativas focales de otras lenguas (p. ej. either y neither).

Con este fin, se han presentado en primer lugar aquellas propiedades distribucionales y combinatorias en las que $O_{1}$ y $n i_{1}$ se alejan de los coordinadores disyuntivos y copulativos simples $(o / y)$. En concreto, se ha mostrado que $O_{1}$ y $n i_{1}$, a diferencia de los coordinadores, pueden estar precedidos por las conjunciones pero e $y$, anteceden inmediatamente al primer coordinando del conjunto, no son recursivos, no aparecen con términos de preposición y son incompatibles con posesivos dentro del mismo SN.

En segundo lugar, se han presentado las pruebas que permiten relacionar $o_{1}$ y $n i_{1}$ con las partículas correlativas y, por extensión, con las partículas focales. En el plano gramatical, $O_{1}$ y $n i_{1}$ exigen la presencia de un correlato formalmente determinado (o lo presuponen en las construcciones truncas con $O_{1}$ ), no son recursivas y se combinan siempre con proyecciones máximas o sintagmas. En lo que se refiere al plano de la pragmática, $O_{1}$ y $n i_{1}$, como las partículas correlativas de otras lenguas, muestran una estrecha relación con las partículas focales. Así, $O_{1}$ y $n i_{1}$ dotan de rasgos prosódicos especiales a la oración (abren una curva tonal de anticadencia o semianticadencia que se cierra con el correlato), condicionan la codificación del tópico y expresan exclusión entre distintas alternativas. $O_{1}$ expresa disyunción exclusiva con preferencia por el primer miembro del conjunto, mientras que $n i_{1}$, que también expresa exclusión, subraya el carácter cerrado del conjunto de ni... ni y enfatiza la negación de entidades que son esperables o conocidas en el discurso o en el contexto. Asimismo, entre sus funciones pragmáticas, $n i_{\perp}$ permite interpretar el alcance de la negación sobre los coordinandos de la correlación en aquellos casos en los que la partícula es facultativa.

Por último, en este artículo se ha señalado la conveniencia de tratar las partículas $\mathrm{O}_{1}$ y $n i_{1}$ como operadores con repercusión semántica y pragmática sobre la oración.

\section{Bibliografía citada}

Alarcos, Emilio, 1994: Gramática de la lengua española, Madrid: Espasa Calpe.

Aldaber, Majed Hassan M. 2018: La sintaxis de la coordinación distributiva. Tesis doctoral, Universidad Complutense de Madrid.

Bosque, Ignacio, 1994: "La negación y el principio de las categorías vacías" en Violeta Demonte (comp.): Gramática española, México, D. F.: Publicaciones de la Nueva Revista de Filología Hispánica, 167-199.

CAmacho, Jorge, 1999: “La coordinación” en Ignacio Bosque y Violeta Demonte (dirs.): Gramática descriptiva de la lengua española, vol. 2, Madrid: Espasa, 2635-2694. 
CAMACHo, Jorge, 2003: The structure of coordination: conjunction and agreement phenomena in Spanish and other languages, Dordrecht: Kluwer Academic Publishers.

CONTI, Carmen, 2014: "Hacia una caracterización gramatical de las relaciones interclausales en español”, Verba 41, 25-49.

ContI, Carmen, 2016: "Coordinación y cláusulas adversativas: problemas clasificatorios y propuesta de análisis”, Revista Española de Lingüística 46 (2), 7-30.

ContI, Carmen, 2018a: "Disjunctive clauses with o... o 'either... or' in Spanish and clausal cosubordination", Folia Linguistica 52 (1), 75-105.

ContI, Carmen, 2018b: "Construcciones consecutivas ponderativas en español: focalización, correlativos y correlatos adjuntos”, Zeitschrift für romanische Philologie 134 (3), 1-24.

Dık, Simon, [1968] 1972: Coordination. Its implications for the theory of general linguistics, Amsterdam: North-Holland.

Férr, Caroline, 2013: "Focus as prosodic alignment", Natural Language \& Linguistic Theory 31, 683-734.

Fornés, Mercedes, 1994: "Sobre el paradigma de formas que expresan disyunción en español”, Anuario de Estudios Filológicos 17, 133-150.

Fonnés, Mercedes, 1996: La interacción de factores textuales: una interpretación de la denominada "coordinación distributiva", Pamplona: EDUNSA.

Franchinl, Enzo, 1986: Las condiciones gramaticales de la coordinación copulativa en español, Bern: A. Francke AG Verlag.

Fukasawa, Mitsuyo, 1985: "La coordinación disyuntiva en español: aspecto sincrónico", RILCE: Revista de Filología Hispánica 1 (1), 47-81.

Hendriks, Petra, 2001: "Initial coordination and the law of coordination of likes" en Ton van der Wouden y Hans Broekruis (eds.): Linguistics in the Netherland, Amsterdam: John Benjamins, 127-138.

Hendriks, Petra, 2003: "Either as a focus particle” [http://www.let.rug.nl/hendriks/papers/ either03.pdf, última consulta: 24-09-2018].

Hendriks, Petra, 2004: "Either, both and neither in coordinate structures" en Alice ter Meulen y Abraham Werner (eds.): The Composition of Meaning: From Lexeme to Discourse, Amsterdam: John Benjamins, 115-138. 
Hengeveld, Kees, y J. Lachlan Mackenzie, 2008: Functional discourse grammar. A typologicallybased theory of language structure, Oxford: Oxford University Press.

Jiménez JulıÁ, Tomás, 1984: “La llamada coordinación negativa en español”, Verba 11, 21-244.

JiméneZ Juliá, Tomás, 1986: “Disyunción exclusiva e inclusiva en español”, Verba 13, 163-180.

Jiménez Julia, Tomás, 1995: La coordinación en español. Problemas teóricos y descriptivos, Santiago de Compostela: Universidad de Santiago de Compostela.

JohANNESSEN, Janne Bondi, 2005: "The syntax of correlative adverbs", Lingua 115, 419-443.

KönIG, Ekkehard, 1991: The meaning of focus particles: A comparative perspective, London/ New York: Routledge.

LARSON, Richard, 1985: "On the syntax of disjunction scope”, Natural Language \& Linguistic Theory 3, 217-264.

LIPTÁk, Anikó (ed.), 2009: Correlatives cross-linguistically, Amsterdam/Philadelphia: John Benjamins.

LIPTAK, Anikó, 2009: "The landscape of correlatives: An empirical and analytical survey” en Anikó LIPTAK (ed.): Correlatives cross-linguistically, Amsterdam; Philadelphia: John Benjamins, 1-48.

Martínez, José A., 1994: La oración compuesta y compleja, Madrid: Arco Libros.

Myre, Annette Maria Jørgensen, 1992: "Reflexiones sobre las conjunciones en las construcciones distributivas" en Antonio Vilanova (coord.): Actas del X Congreso de la Asociación Internacional de Hispanistas, Barcelona 21-26 de agosto de 1989, vol. 4, Barcelona: Promociones y Publicaciones Universitarias, 1263-1272.

Myre, Annette Maria Jørgensen, 1998: Las oraciones coordinadas distributivas. Los mecanismos de las oraciones coordinadas distributivas y sus correlatos, Bergen: Bergen University.

Real Academia Española, 1973: Esbozo de una nueva gramática de la lengua española, Madrid: Espasa-Calpe.

Real Academia Española, 2013-presente: Corpus del español del siglo XXI (CORPES) [disponible en http://www.rae.es].

Real Academia Española y Asociación de Academias de la Lengua Española, 2009: Nueva Gramática de la Lengua Española, vol. II, Madrid: Espasa. 
SÁnCHEZ, Cristina, 1999: "Los cuantificadores: clases de cuantificadores y estructuras cuantificativas” en Ignacio Bosque y Violeta Demonte (dirs.): Gramática descriptiva de la lengua española, vol. 1, Madrid: Espasa, 1129-1188.

Sudhoff, Stefan, 2010: Focus particles in German: Syntax, prosody, and information structure, Amsterdam/Philadelphia: John Benjamins.

Schwarz, Bernhard, 1999: "On the syntax of either... or", Natural Language \& Linguistic Theory 17, 339-370.

VAN VauIN, Jr., Robert D., 2005: Exploring the Syntax-Semantics Interface, Cambridge: Cambridge University Press.

Van Valin, Jr., Robert D., y Randy LAPolla, 1997: Syntax: Structure, Meaning and Function, Cambridge: Cambridge University Press. 